\title{
Treatments of osteoporosis increase bone material strength index in patients with low bone mass
}

\author{
M. Schoeb ${ }^{1}$ (D) F. Malgo ${ }^{1}$ (D) J. J. M. Peeters ${ }^{1}$ (D) E. M. Winter ${ }^{1}$ (D) - S. E. Papapoulos ${ }^{1} \cdot$ N. M. Appelman-Dijkstra $^{1}$ (D)
}

Received: 9 December 2019 / Accepted: 3 March 2020 / Published online: 8 April 2020

(C) The Author(s) 2020

\begin{abstract}
Summary Effects on bone material properties of two-year antiosteoporotic treatment were assessed using in vivo impact microindentation (IMI) in patients with low bone mineral density (BMD) values. Antiresorptive treatment, in contrast to vitamin $\mathrm{D} \pm$ calcium treatment alone, induced BMD-independent increases in bone material strength index, measured by IMI, the magnitude of which depended on pretreatment values.

Introduction Bone material strength index (BMSi), measured by IMI in vivo, is reduced in patients with fragility fractures, but there is no information about changes in values during long-term therapy. In the present study, we assessed changes in BMSi in patients receiving antiosteoporotic treatments for periods longer than 12 months.

Methods We included treatment-naive patients with low bone mass who had a BMSi measurement with OsteoProbe® at presentation and consented to a repeat measurement after treatment.

Results We studied 54 patients ( 34 women), median age 58 years, of whom 30 were treated with bisphosphonates or denosumab (treatment group) and 24 with vitamin $\mathrm{D} \pm$ calcium alone (control group). There were no differences in clinical characteristics between the two groups with the exception of a higher number of previous fragility fractures in the treatment group. Baseline hip BMD and BMSi values were lower in the treatment group. After 23.1 \pm 6.6 months, BMSi increased significantly in the treatment group $(82.4 \pm 4.3$ vs $79.3 \pm 4.1 ; p<0.001)$, but did not change in the control group $(81.5 \pm 5.2$ vs $82.2 \pm 4.1 ; p=0.35)$. Changes in BMSi with antiresorptives were inversely related with baseline values $(r=-0.43 ; p=0.02)$ but not with changes in BMD. Two patients in the control group with large decreases in BMSi values sustained incident fractures.

Conclusion In patients at increased fracture risk, antiresorptive treatments induced BMD-independent increases in BMSi values, the magnitude of which depended on pretreatment values.
\end{abstract}

Keywords Bisphosphonates · Bone material strength index $(\mathrm{BMSi}) \cdot$ Denosumab $\cdot$ Fragility fracture $\cdot$ Impact microindentation · Reference point indentation

\section{Introduction}

Impact microindentation (IMI), a method to assess tissue-level properties of cortical bone in vivo $[1,2]$, is being increasingly used in studies evaluating the contribution of such properties to bone fragility in humans [3-6]. The resistance of cortical bone to indentation, measured as bone material strength index (BMSi), was decreased in individuals with fragility fractures

N. M. Appelman-Dijkstra

n.m.appelman-dijkstra@lumc.nl

1 Center for Bone Quality, Department of Medicine, Division Endocrinology, Leiden University Medical Center,

Leiden, The Netherlands compared with appropriate controls in several studies [7-10]. It is not related to bone mineral density (BMD) values [7-10], but, as recently shown, BMSi most likely assesses subperiosteal bone material properties [11].

Short-term effects of antiosteoporotic treatments on BMSi have been examined in patients initiating glucocorticoid therapy [12]. Glucocorticoids induced rapid decreases of BMSi, while treatments prevented this decline or even increased BMSi values within 7 weeks, up to 20 weeks, with no concurrent changes in BMD values. These results strongly suggested that BMSi could detect early, treatment-induced changes in bone material properties in glucocorticoid-treated patients. In addition, increases in BMSi have been reported in postmenopausal women 3 months after a high impact exercise program [13], and in HIV-infected patients after 1 year 
treatment with antiretroviral agents [14] or following gastric bypass surgery in obese subjects with and without type 2 diabetes [15]. However, the effect of antiosteoporotic treatments on BMSi in patients at increased risk of fractures not receiving glucocorticoids has not been investigated. Moreover, osteoporosis is a chronic disease requiring long-term treatment, and it is currently unknown whether changes in BMSi over time might provide information, additional to those obtained by BMD measurements, in treated individuals.

In the present study, we addressed this question in individuals at increased risk of fractures by measuring BMSi with the handheld IMI device OsteoProbe ${ }^{\circledR}$ before and after treatments given for periods longer than 12 months.

\section{Patients and methods}

\section{Study design}

Observational study evaluating longitudinal changes in BMSi with antiresorptive agents or vitamin $\mathrm{D} \pm$ calcium alone in women and men with low bone mass attending the outpatient clinic of the Center for Bone Quality of the Leiden University Medical Center (LUMC) between March 2015 and September 2018.

\section{Patients}

Included in the study were treatment-naive subjects $\geq 18$ years with osteopenia or osteoporosis who had an IMI measurement at presentation, were followed by one of the authors (NAD) for at least 1 year and consented to a repeat measurement. Exclusion criteria included any treatment affecting bone metabolism during follow-up-except calcium, vitamin D, bisphosphonates or denosumab - a metabolic bone disease other than osteoporosis, immobilization and local pathologies of the tibia or skin at the site of examination.

Treatment with antiresorptive agents (with vitamin $\mathrm{D} \pm$ calcium) or vitamin $\mathrm{D} \pm$ calcium alone was given according to Dutch national guidelines for the management of osteoporosis [16]. Treatment was initiated with oral bisphosphonates (BP) unless patients had epigastric complaints or did not want to receive oral BP; in these patients, iv BP or denosumab (DMAb) were given according to patients' preference and physician's judgement. All patients were followed at regular intervals in the outpatient clinic according to protocols of the Center for Bone Quality for the different treatment regimens.

The study was approved by the Medical Ethical Committee of the LUMC, and written informed consent was obtained from all individuals included in the study.

\section{Methods}

Detailed medical history, clinical risk factors for fracture, fracture history with documentation of sites and dates of occurrence and information about use of medication were collected at baseline and follow-up visits. A fragility fracture was defined as any low-energy fracture, excluding those of the hands, feet and skull. Reasons and time of discontinuation or change to another antiresorptive agent during follow-up were recorded.

\section{Laboratory measurements}

Serum calcium (albumin-corrected), creatinine and alkaline phosphatase were measured by semiautomated techniques. Plasma intact PTH was measured by immulite 2500 (Siemens Diagnostics, Breda, The Netherlands) and serum 25-hydroxyvitamin D (25-OH D) by the 25-OH-vitamin D TOTAL assay (DiaSorin D.A./N.V., Brussels, Belgium).

\section{Bone mineral density}

Areal BMD was measured at the lumbar spine (L1-L4) and the left and right hip by dual-energy X-ray absorptiometry (DXA) with the Hologic QDR 4500 (Hologic Inc., Waltham, MA, USA). Average BMD values of the hip were used in the analysis. NHANES III reference values compatible with reference values of the Dutch population were used to calculate T-scores. Osteopenia and osteoporosis were diagnosed according to $\mathrm{WHO}$ criteria.

\section{Vertebral fracture assessment}

Spine radiographs for the detection of vertebral deformities were performed at baseline in all patients. During follow-up, radiographs were only performed if there was loss of height $>$ $3 \mathrm{~cm}$ or clinical complaints. Radiographs were independently evaluated by two of the authors (NAD and MS) using the semiquantitative method of Genant et al. [17], and only fractures grade 2 or higher were considered in the analysis.

\section{Bone material strength index}

BMSi was measured in all patients by IMI using the handheld microindenter device (OsteoProbe ${ }^{\circledR}$ RUO, Active Life Scientific, CA, USA) at the midshaft of the tibia. Measurements were performed by two experienced operators (NAD and FM) according to our previously published protocol $[7,8,18,19]$. The patient was placed in a decubitus supine position with the tibia in external rotation. The measurement site was defined as the mean distance between the medial malleolus and the distal apex of the patella. After disinfection and local anaesthesia of the skin and periosteum with 
lidocaine $1 \%$, the test probe was gently inserted in the skin until the bone surface was reached. It was ensured that the test probe was always perpendicular to the bone surface during measurements. The operator was not allowed to check the measurements on the computer screen before these were classified as "well performed", "adequate" or "poorly performed". Measurements were classified as "well performed" when the test probe was exactly perpendicular to the bone surface, as "adequate" when the test probe was within acceptable deviation from the bone surface [2] and as "poorly performed" when the operator judged that the test probe was not appropriately placed. "Poorly performed" measurements are usually due to slipping of the test probe, moving of the subject's leg, indenting the same spot twice, or failure to place the device perpendicularly to the bone surface and were manually discarded. In addition, the first measurement was systematically discarded since there is often inadequate penetration of the probe through the periosteum. After at least five adequate measurements (range 6 to 15 , mean $10.0 \pm 1.8$ measurements), five additional measurements were performed on a polymethylmethacrylate (PMMA) calibration phantom. BMSi was calculated by the computer software. The operator was not blinded to the patient's treatment, but was blinded to baseline BMSi results, and classification of measurements was done before checking the results on the computer screen. The intraobserver coefficient of variation $(\mathrm{CV})$ was $2.2 \%$, and the interobserver $\mathrm{CV}$ was $1.6 \%$.

\section{Statistical analysis}

Results are presented as mean \pm SD unless otherwise stated. Descriptive statistics were used to describe clinical and laboratory parameters. Between-group differences in baseline characteristics were assessed using a Student's $t$ test or Mann-Whitney $U$ test and chi-square test or Fisher's exact test for normally and not normally distributed continuous and for categorical variables, respectively. Within-group changes in BMSi and BMD were assessed using a paired $t$ test. Normality of the distribution of BMSi and BMD was checked by a Kolmogorov-Smirnov test and visually with histograms. Between-group differences in \% changes from baseline to follow-up measurements were assessed by a Student's $t$ test. ANCOVA models with \% change in BMSi as outcome variable, adjusted for baseline BMSi, BMD and fragility fracture, were used to compare \% change in BMSi between groups. Correlations between BMSi and BMD values and between $\%$ changes in BMSi and \% changes in BMD or duration of treatment were examined by a Pearson's test. To determine independent effects of factors possibly influencing changes in BMSi, a multiple linear regression analysis was used. Age, gender, fragility fracture, baseline BMSi, treatment duration as well as examiner of the first and the second IMI measurement, respectively, were included in the model. A power calculation was performed using a difference of $3.1 \%$ in BMSi values. This difference was previously found between patients with and patients without fragility fractures (BMSi $79.9 \pm 0.6$ vs $82.4 \pm 1.0$ ) and considered to be clinically relevant [7]. The sample size to detect this difference in BMSi from baseline to follow-up with a standard deviation of 5 and power of 0.8 at a significance level of 0.05 was calculated to be 24 per group. All analyses were performed using SPSS software for Windows (version 25.0; SPSS Inc., Chicago, IL, USA). A $p$ value < 0.05 was considered to be statistically significant. Graphs were constructed with Graphpad Prism (version 8.0; Graphpad Software Inc., La Jolla, CA, USA).

\section{Results}

Fifty-four patients were included in the study (Fig. 1). These were 34 women and 20 men with median age 58.0 years (IQR 48.5-63.3 years). Thirty-one patients had osteoporosis (57.4\%) and 23 had osteopenia (42.6\%); 23 patients ( 7 men) had sustained one or more fragility fractures (11 vertebral, 2 hip, 14 non-hip/non-vertebral) at the time of the first IMI measurement (Table 1). Mean time to second measurement was $23.1 \pm 6.6$ months.

Baseline characteristics of patients who were excluded from the study, either because they were referred to their general practitioners, were followed by other physicians in our centre or were lost to follow-up (Fig. 1), were not different from those of the included patients; age $56.2 \pm 14.2$ vs $55.3 \pm$ 13.0 years, $p=0.637$; female gender $59.5 \%$ vs $63.0 \%, p=$ 0.665 ; prevalent fragility fractures $47.7 \%$ vs $42.6 \%, p=$ 0.500 ; BMSi $80.7 \pm 5.6$ vs $80.6 \pm 4.3, p=0.894$.

Of the 54 patients included in the analysis, 30 started antiresorptive therapy after a median of 4 days following the first IMI measurement (treatment group); 23 patients received BPs (16 oral, 7 iv) and 7 DMAb. Twenty-four patients received vitamin $\mathrm{D} \pm$ calcium alone (control group) (Table 1). Patients in the treatment group had sustained significantly more fragility fractures compared with those of the control group ( 25 vs $4, p<0.01$ ), due mainly to the higher prevalence of vertebral fractures in the former group (14 vs $0, p<0.01)$.

\section{Bone mineral density}

In the treatment group, 20 patients had osteoporosis (T-score $\leq$ -2.5 ) and 10 had osteopenia (T-score between -2.5 and $-1.0)$; in the control group, 11 patients had osteoporosis and 13 had osteopenia ( $p=0.124$ between the two groups). Lumbar spine (LS) BMD was lower in the treatment group, but the difference between the two groups was not significant $\left(0.81 \pm 0.08\right.$ vs $\left.0.86 \pm 0.10 \mathrm{~g} / \mathrm{cm}^{2}, p=0.06\right)$; femoral neck (FN) BMD and total hip (TH) BMD were significantly lower 


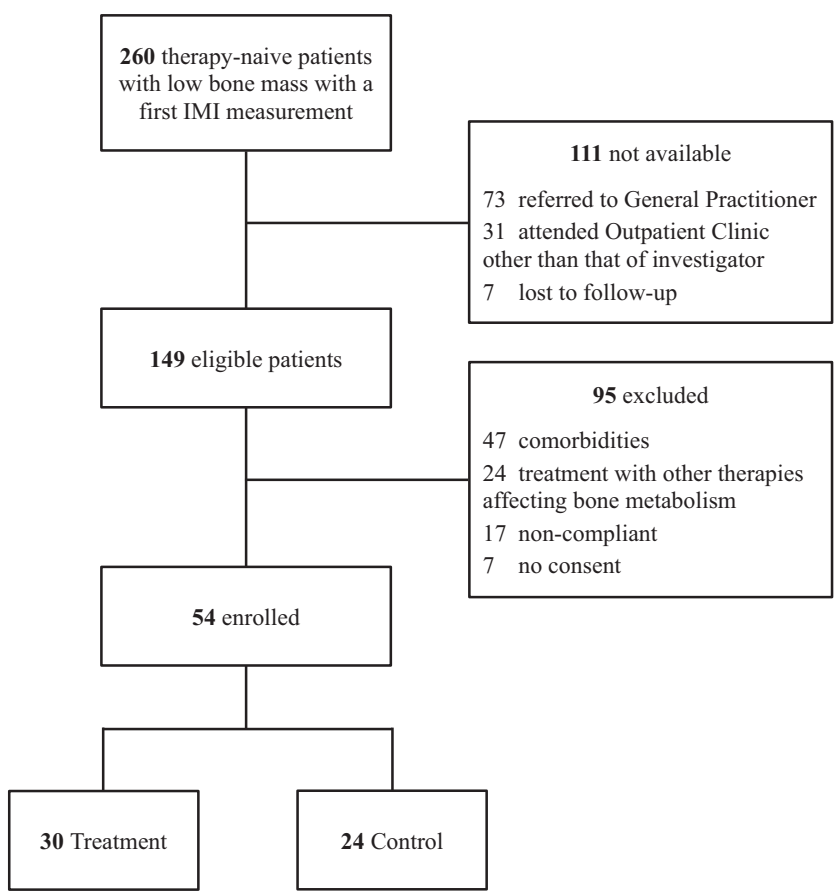

in the treatment group (FN $0.66 \pm 0.09$ vs $0.70 \pm$ $0.09 \mathrm{~g} / \mathrm{cm}^{2}, p=0.041 ;$ TH $0.77 \pm 0.10$ vs $0.84 \pm$ $\left.0.12 \mathrm{~g} / \mathrm{cm}^{2}, p=0.016\right)$.

Antiresorptive treatment was associated with significant increases in LS-BMD $(6.3 \pm 5.3 \%)$ and TH-BMD $(1.1 \pm$ $2.9 \%$ ) but not in FN-BMD (1.2 $\pm 3.6 \%)$ (Fig. 2). In the control group, LS-BMD did not change significantly during followup $(-1.3 \pm 3.6 \%$,), while hip BMD decreased significantly at both measured sites (FN-BMD $-2.7 \pm 4.6 \%$, TH-BMD -2.4 $\pm 2.9 \%$ ) (Fig. 2).

\section{Bone material strength index}

Consistent with our previous studies, baseline BMSi values of all studied patients were not associated with baseline BMD values at any site (LS $r=-0.072, p=$ 0.617 ; FN $r=0.067, p=0.631$; TH $r=0.048, p=0.728$ ) and were significantly lower in patients with fragility fractures compared with those without $(78.5 \pm 3.0$ vs $82.1 \pm 4.5, p=0.002)$.

Fig. 1 Patient flowchart

Table 1 Baseline characteristics of 54 patients

\begin{tabular}{|c|c|c|c|c|}
\hline Characteristics & All patients & Treatment & Control & $p$ value \\
\hline$n$ & 54 & 30 & 24 & \\
\hline Age, years & $58.0 \pm 1.8$ & $59.0 \pm 1.8$ & $55.0 \pm 3.2$ & 0.16 \\
\hline Male/female & $20 / 34$ & $8 / 22$ & $12 / 12$ & 0.11 \\
\hline BMI, $\mathrm{kg} / \mathrm{m}^{2}$ & $24.2 \pm 3.6$ & $24.0 \pm 3.4$ & $24.5 \pm 4.0$ & 0.67 \\
\hline Smoking, $n$ & 7 & 6 & 1 & 0.12 \\
\hline Alcohol use $\geq 3 \mathrm{U} / \mathrm{d}, n$ & 5 & 2 & 3 & 0.65 \\
\hline Previous fragility fracture, $n(\%)$ & $23(42.6)$ & $19(63.3)$ & $4(16.7)$ & 0.001 \\
\hline Hip fracture, $n(\%)$ & $2(3.7)$ & $2(6.7)$ & $0(0.0)$ & 0.50 \\
\hline NHNV fracture, $n(\%)$ & $14(25.9)$ & $10(33.3)$ & $4(16.7)$ & 0.17 \\
\hline Vertebral fracture, $n(\%)$ & $11(20.4)$ & $11(40.0)$ & $0(0.0)$ & 0.001 \\
\hline Calcium $^{\mathrm{a}}, \mathrm{mmol} / \mathrm{L}$ & $2.32 \pm 0.08$ & $2.34 \pm 0.08$ & $2.31 \pm 0.08$ & 0.11 \\
\hline Creatinine $^{\mathrm{b}}$, umol/L & $76.1 \pm 15.0$ & $74.0 \pm 13.8$ & $78.8 \pm 16.2$ & 0.24 \\
\hline $25-\mathrm{OH} \mathrm{D} \mathrm{D}^{\mathrm{c}}, \mathrm{nmol} / \mathrm{L}$ & $80.9 \pm 27.7$ & $78.7 \pm 29.0$ & $83.6 \pm 26.4$ & 0.52 \\
\hline $\mathrm{PTH}^{\mathrm{d}}, \mathrm{pmol} / \mathrm{L}$ & $3.5 \pm 1.3$ & $3.6 \pm 1.6$ & $3.2 \pm 0.9$ & 0.13 \\
\hline LS-BMD T-score & $-2.1 \pm 0.8$ & $-2.3 \pm 0.7$ & $-1.9 \pm 0.9$ & 0.10 \\
\hline FN-BMD T-score & $-1.7 \pm 0.7$ & $-1.9 \pm 0.7$ & $-1.5 \pm 0.6$ & 0.08 \\
\hline TH-BMD T-score & $-1.3 \pm 0.7$ & $-1.5 \pm 0.6$ & $-1.1 \pm 0.8$ & 0.026 \\
\hline $\mathrm{BMSi}$ & $80.6 \pm 4.3$ & $79.3 \pm 4.1$ & $82.2 \pm 4.1$ & 0.014 \\
\hline
\end{tabular}

$N H N V$, Non-hip/non-vertebral; $B M D$, bone mineral density; $L S$, lumbar spine; $F N$, femoral neck; $T H$, total hip; $B M S i$, bone material strength index. Values are expressed as mean \pm SD. Age is expressed as median \pm SEM. $p$ values are displayed for treatment vs control

${ }^{\mathrm{a}}$ Calcium (albumin-corrected) reference range, 2.15-2.55 mmol/L

${ }^{\mathrm{b}}$ Creatinine reference range, 64-104 umol/L for males; 49-90 umol/L for females

${ }^{\mathrm{c}} 25-\mathrm{OH}$ vitamin D reference range, 50-250 nmol/L

${ }^{\mathrm{d}} \mathrm{PTH}$ reference range, $0.7-8.0 \mathrm{pmol} / \mathrm{L}$ 
Fig. 2 Mean $( \pm \mathrm{SD})$ percentage changes in bone mineral density (BMD) at follow-up compared with baseline in the treatment (black bars) and control group (grey bars) at the lumbar spine (LS), femoral neck $(\mathrm{FN})$ and total hip (TH). *Significantly different from baseline, $p<0.05$ and $* * p<0.001$

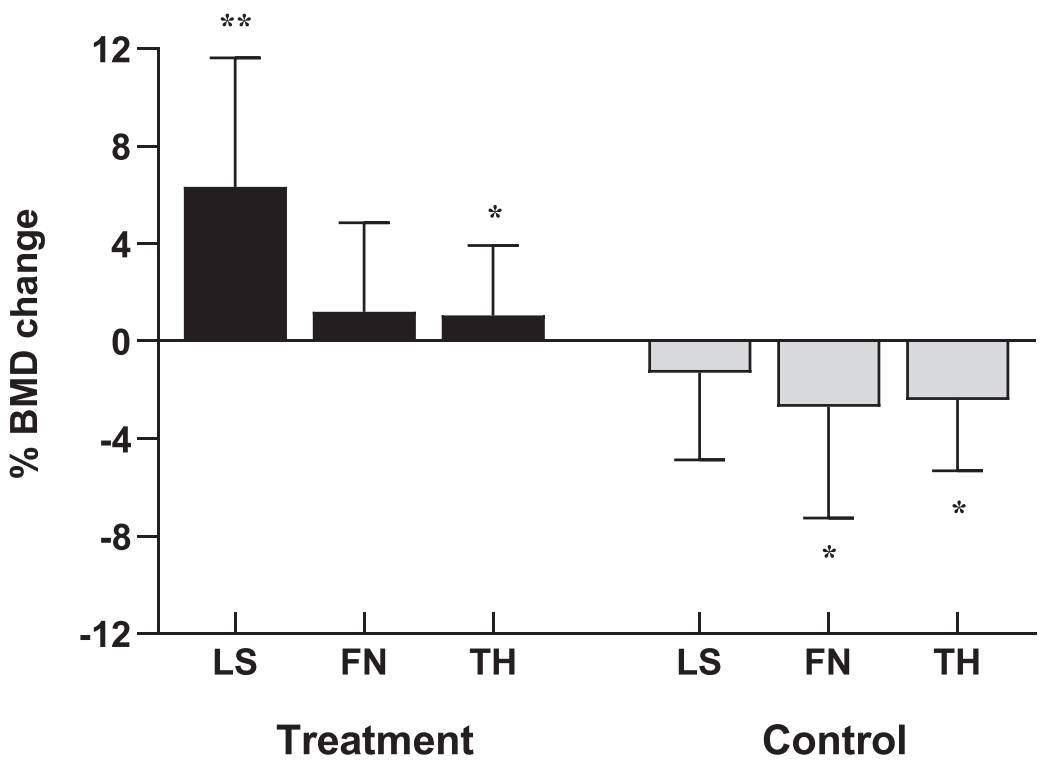

\section{Changes during follow-up}

Baseline BMSi values of the treatment group were significantly lower than those of the control group (Table 1) and increased significantly by $4.0 \pm 5.2 \%$ to values similar to those of the control group at baseline (from 79.3 \pm 4.1 to $82.4 \pm 4.3, p<0.001$ ) (Fig. 3). The increase in BMSi with antiresorptive treatment was inversely related with baseline BMSi $(r=-0.432, p=0.017)$ (Fig. 4) but not with the duration of treatment $(r=0.089 p=0.639)$ or with the BMD changes (LS $r=-0.076, p=0.712$; FN $r=$ $0.266, p=0.155$; TH $r=-0.031, p=0.870)$. In contrast, in the control group, BMSi values decreased during the period of observation but not significantly (from $82.2 \pm 4.1$ to $81.5 \pm 5.2, p=0.353 ;-0.7 \pm 3.9 \%$ ) (Fig. 3 ). The difference in \% BMSi changes between the two groups was significant, $p<0.001$ (also after adjusting for baseline BMSi $(p=0.004)$, baseline BMD $(p=0.007)$ and prevalent fragility fractures $(p=0.016))$.

In both BP- and DMAb-treated patients for the whole observation period, BMSi increased significantly (BP from 80.1 \pm 4.6 to $82.5 \pm 4.4(3.1 \pm 5.6 \%), p=0.037$; DMAb from 78.1 \pm 3.1 to $84.2 \pm 4.3(7.8 \pm 3.1 \%), p=0.001)$. The percentage increase in BMSi was higher in the DMAb-treated patients ( $p=0.014)$, but after adjusting for baseline BMSi, the difference between the two treatments was not significant anymore $(7.1 \pm 4.8 \%$ vs $3.4 \pm 4.7 \%, p=0.104)$. Numbers however were small.
Fig. 3 Bone material strength index (BMSi) in patients with antiresorptive treatment (treatment) and those without antiresorptive treatment (control) at baseline (BL) and follow-up (FU). Data are shown in boxwhisker plots and statistical differences are displayed for BMSi. Boxes indicate median and interquartile range. Bars indicate minimum and maximum values. $* * p<0.001$

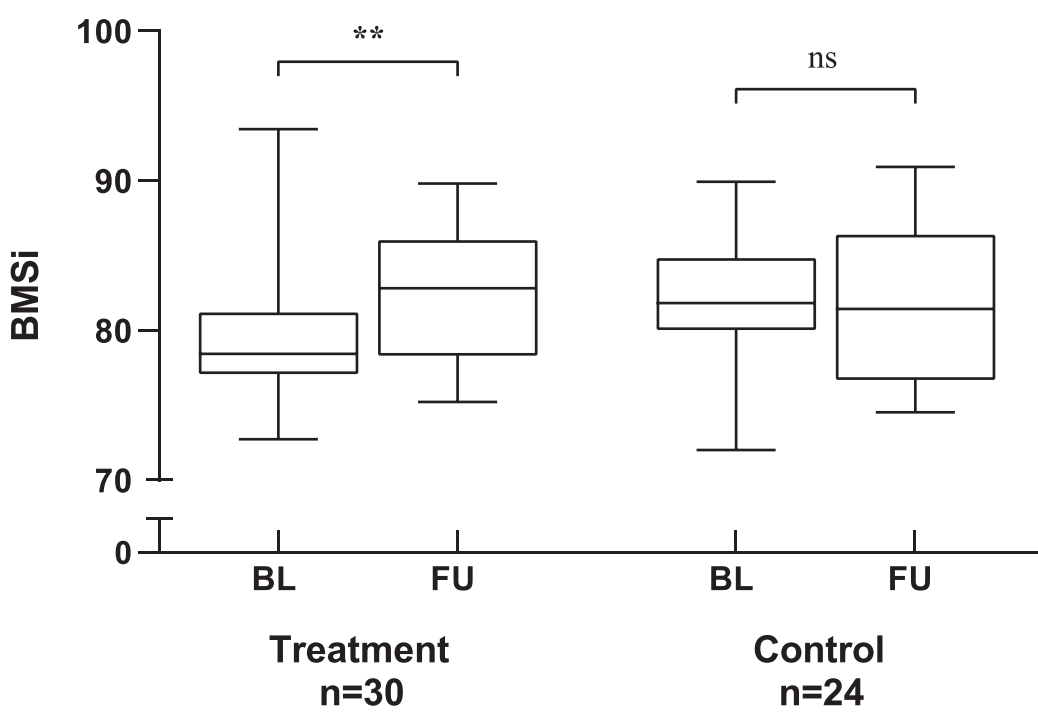




\section{Fractures}

During the period of observation, there were no fragility fractures in the treatment group while two patients of the control group sustained fragility fractures. In the first patient, a decline of BMSi by $8.5 \%$ was documented 17 months before a humerus fracture; the corresponding decreases of LS-, FN- and THBMD were $-2.2 \%,-1.3 \%$ and $-2.7 \%$ to T-scores of -1.5 , -0.9 and -0.4 , respectively. New assessment revealed no causes of secondary osteoporosis. The second patient sustained a Weber B ankle fracture 18 months after the second measurement that showed an $8.9 \%$ decline of BMSi; the corresponding decreases of LS-, FN- and TH-BMD were $-4.8 \%,-7.0 \%$ and $-4.3 \%$ to $\mathrm{T}$-scores of $-2.2,-1.7$ and -0.4 , respectively.

\section{Discussion}

This study demonstrates that treatment with BPs and DMAb, given for a mean period of 2 years, increases BMSi values, measured by impact microindentation, in patients with low bone mass and increased risk of fracture. The values of BMSi reached with treatment were not different from baseline values of the patients of the control group as well as from those previously reported in patients with low bone mass without fractures $[7,8]$. The magnitude of these changes was, however, different among treated patients and depended on baseline values with larger increases observed in the patients with lower baseline values. Our results are, therefore, in agreement with those obtained after short-term treatment of patients receiving glucocorticoids [12]. The pattern of BMSi changes by antiresorptive therapy was further similar to that observed after treatment of HIV-infected patients with antiretroviral agents or following gastric bypass

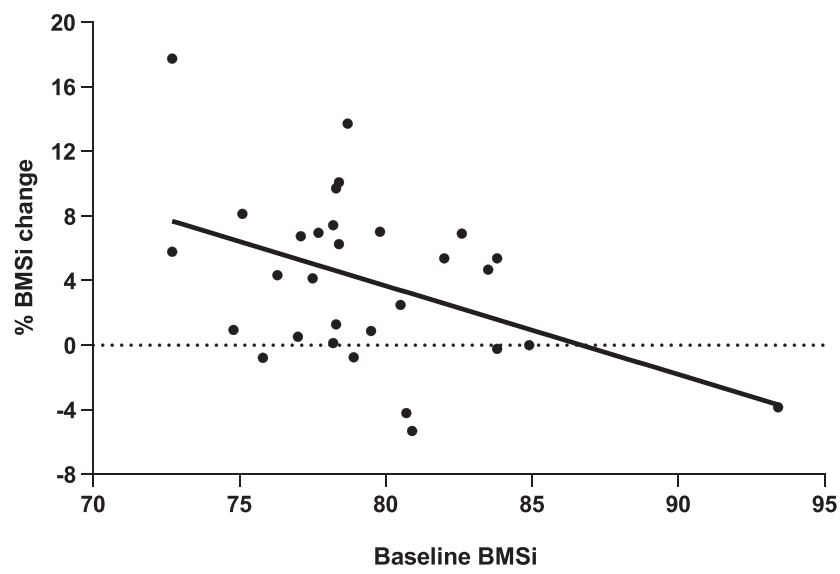

Fig. 4 Relationship between bone material strength index (BMSi) at baseline and \% BMSi change from baseline to follow-up in 30 patients treated with antiresorptive agents. $r=-0.432, p=0.017$. Absence of change in BMSi is indicated by the dashed line surgery in obese subjects with and without type 2 diabetes $[14,15]$.

The increases in BMSi values observed in our and other studies $[12,14,15]$ were not related with changes in BMD illustrating, in a different setting, the independence of measurements of bone material properties from those of mineral density as also reported previously in cross-sectional studies [7-10, 20-28]. We recently confirmed the lack of a relationship between BMSi and BMD by direct measurements of bone mineral density distribution (BMDD) by quantitative backscattered electron imaging (qBEI) in whole bone tissue in iliac crest bone biopsies obtained concurrently with BMSi measurements in patients with a wide range of BMD [11].

In the present study, patients treated with antiresorptive agents had lower baseline BMSi values and a higher number of fragility fractures than those treated with vitamin $\mathrm{D} \pm$ calcium alone. Notably, BMSi was not considered in treatment decision that was based on conventional tools to assess fracture risk. Guidelines for use of BMSi measurements by OsteoProbe ${ }^{\circledR}$ in clinical practice are not yet available, and their value is investigated in research settings, as in our study. Results, however, strengthen the notion that measurements of BMSi may be a useful addition to currently available tools for the assessment of fracture risk.

The results of our study and that of Mellibovsky et al. in glucocorticoid-treated patients [12] demonstrate that BMSi, measured by IMI, increases by treatments of osteoporosis. We show, in addition, that these increases are not related to treatment duration and appear to have a plateau which is essentially not different from normal values and might represent the maximum value that can be achieved with treatment. This plateau was reached with both DMAb and BP, and the significantly higher increases in BMSi values with DMAb are likely due to the lower baseline BMSi values of patients treated with this more potent agent, but numbers were small.

The mechanism responsible for the increase in BMSi by antiresorptives has not yet been established. We recently showed that BMSi measured by OsteoProbe ${ }^{\circledR}$ is significantly associated with bone material properties, measured by Raman spectroscopy, of subperiosteal bone in patients with different skeletal disorders and a wide range of BMD, with or without fractures [11]. We have now extended this study, and we measured material properties of the whole cortex of these biopsies by Fourier transform infrared imaging (FTIRI), a spectroscopic method more suitable for the measurement of compositional bone changes in large areas (manuscript in preparation). In this analysis, BMSi values were strongly associated, $p<0.01$, with the mineral to matrix ratio (MM), the most widely measured bone material property, that, differently from other measurements of mineral density, directly measures and accounts for the amount of organic matrix in the volume analysed [29]. In rodents, MM correlates with ash weight 
and is directly proportional to bending stiffness and failure moment being superior to total mineral density, measured by micro-CT, in predicting bone strength [30,31]. Bisphosphonates and hormone replacement therapy given for 1 to 3 years to animals or humans increased significantly spectroscopically measured MM [32-35]. Moreover, inhibition of receptor activator of nuclear factor kappa-B ligand (RANKL) by osteoprotegerin (OPG) significantly increased MM in genetically modified ovariectomized mice to levels of wild-type animals (E. Paschalis personal communication). It may, therefore, be that BMSi measurements capture the observed increases in MM by antiresorptive therapies. Notably, prolongation of treatment with bisphosphonate, as in the FLEX study with alendronate, is not associated with continuous increase in MM [36] indicating that the amount of mineral taken up by the matrix in non-pathological mineralisation is limited, or self-regulated; this finding is similar to the observed plateau of bone matrix mineralisation between 5 and 10 years treatment of patients with osteoporosis with denosumab [37] and may explain the lack of further increases in BMSi values when these reach normal levels shown in our study.

Notably, patients on antiresorptive treatments in our study sustained no fractures, whereas fractures occurred only in two patients in the control group. These two patients showed significant decreases in BMSi values $(-8.5 \%$ and $-8.9 \%)$ during follow-up, whereas the corresponding decreases in BMD were variable and ranged between $-1.3 \%$ and - 7.0\%. Moreover, in a cross-sectional study of postmenopausal women on long-term treatment with bisphosphonates (4 to 14 years) BMSi values were significantly lower in women with incident fractures compared with those without fractures [28]. Thus, IMI measurements may not only be a useful, complementary investigation in the assessment of fracture risk but may also provide additional long-term information about the effects of treatments on bone material properties and their association to bone fragility. This needs, however, to be confirmed in appropriately designed studies.

A limitation of the study is the inclusion of only patients followed by one of the authors. However, baseline characteristics of patients who had a baseline measurement but were not included in the study did not differ from those of patients reported here and treatment protocols were the same. Moreover, all measurements were performed by two experienced investigators that minimized intra- and interobserver variability, a known limitation of the application of IMI technique $[1,2,6]$.

In summary, our study, the first to investigate longitudinal changes in BMSi with antiosteoporotic treatments in patients with low bone mass and increased fracture risk, demonstrates that IMI can capture BMD-independent, treatment-induced increases in BMSi, the magnitude of which depends on pretreatment values.

Acknowledgements The authors thank R. Tsonaka, $\mathrm{PhD}$, Department of Medical Statistics and Bioinformatics, Leiden University Medical Center, for her help in the statistical analysis of the results, and P. Beckers, nurse practitioner of the Center for Bone Quality, Leiden University Medical Center, for her help in the study.

Funding information MS is supported by a clinical research fellowship of the European Calcified Tissue Society (ECTS).

\section{Compliance with ethical standards}

Conflict of interest Manuela Schoeb, Frank Malgo, Joséphine J.M. Peeters and Elizabeth M. Winter declare that they have no conflict of interest. Socrates E. Papapoulos and Natasha M. Appelman-Dijkstra are unpaid members of the Scientific Board of Active Life Scientific, manufacturer of OsteoProbe®

Statement of human rights The study was approved by the Medical Ethical Committee of the LUMC. All procedures performed were in accordance with the ethical standards of the institutional research committee and with the 1964 Helsinki declaration and its later amendments or comparable ethical standards.

Informed consent Written informed consent was obtained from all individual participants included in the study.

Open Access This article is licensed under a Creative Commons Attribution-NonCommercial 4.0 International License, which permits any non-commercial use, sharing, adaptation, distribution and reproduction in any medium or format, as long as you give appropriate credit to the original author(s) and the source, provide a link to the Creative Commons licence, and indicate if changes were made. The images or other third party material in this article are included in the article's Creative Commons licence, unless indicated otherwise in a credit line to the material. If material is not included in the article's Creative Commons licence and your intended use is not permitted by statutory regulation or exceeds the permitted use, you will need to obtain permission directly from the copyright holder. To view a copy of this licence, visit http:// creativecommons.org/licenses/by-nc/4.0/.

\section{References}

1. Diez-Perez A, Bouxsein ML, Eriksen EF, Khosla S, Nyman JS, Papapoulos S et al (2016) Technical note: recommendations for a standard procedure to assess cortical bone at the tissue-level in vivo using impact microindentation. Bone Rep 5:181-185

2. Bridges D, Randall C, Hansma PK (2012) A new device for performing reference point indentation without a reference probe. Rev Sci Instrum 83(4):044301

3. Herrera S, Diez-Perez A (2017) Clinical experience with microindentation in vivo in humans. Bone. 95:175-182

4. Allen MR, McNerny EM, Organ JM, Wallace JM (2015) True gold or pyrite: a review of reference point indentation for assessing bone mechanical properties in vivo. J Bone Miner Res 30(9):1539-1550

5. Diez-Perez A, Farr J (2018) Reference point indentation. In: Bilezikian JP (ed) Primer on the metabolic bone diseases and disorders of mineral metabolism, 9th edn. Wiley, pp 287-292 
6. Schoeb M, Hamdy NAT, Malgo F, Winter EM, Appelman-Dijkstra NM (2020) Added value of impact microindentation in the evaluation of bone fragility: a systematic review of the literature. Front Endocrinol 11(15)

7. Malgo F, Hamdy NA, Papapoulos SE, Appelman-Dijkstra NM (2015) Bone material strength as measured by microindentation in vivo is decreased in patients with fragility fractures independently of bone mineral density. J Clin Endocrinol Metab 100(5):20392045

8. Malgo F, Hamdy NAT, Papapoulos SE, Appelman-Dijkstra NM (2017) Bone material strength index as measured by impact microindentation is low in patients with fractures irrespective of fracture site. Osteoporos Int 28(8):2433-2437

9. Rufus-Membere P, Holloway-Kew KL, Diez-Perez A, Kotowicz MA, Pasco JA (2019) Associations between bone impact microindentation and clinical risk factors for fracture. Endocrinology. en.2019-00415

10. Sosa DD, Eriksen EF (2017) Reduced bone material strength is associated with increased risk and severity of osteoporotic fractures. An impact microindentation study. Calcif Tissue Int 101(1):34-42

11. Rokidi S, Bravenboer N, Gamsjaeger S, Misof B, Blouin S, Chavassieux $P$ et al (2020) Impact microindentation assesses subperiosteal bone material properties in humans. Bone. 131: 115110

12. Mellibovsky L, Prieto-Alhambra D, Mellibovsky F, GuerriFernandez R, Nogues X, Randall C et al (2015) Bone tissue properties measurement by reference point indentation in glucocorticoid-induced osteoporosis. J Bone Miner Res 30(9): 1651-1656

13. Sundh D, Nilsson M, Zoulakis M, Pasco C, Yilmaz M, Kazakia GJ, Hellgren M, Lorentzon M (2018) High-impact mechanical loading increases bone material strength in postmenopausal women-a 3month intervention study. J Bone Miner Res 33(7):1242-1251

14. Guerri-Fernandez R, Lerma-Chippirraz E, Fernandez Marron A, Garcia-Giralt N, Villar-Garcia J, Soldado-Folgado J et al (2018) Bone density, microarchitecture, and tissue quality after 1 year of treatment with tenofovir disoproxil fumarate. Aids. 32(7):913-920

15. Blom-Hogestol IK, Mala T, Kristinsson JA, Brunborg C, Gulseth HL, Eriksen EF (2019) Changes in bone quality after Roux-en-Y gastric bypass: a prospective cohort study in subjects with and without type 2 diabetes. Bone. 130:115069

16. CBO. Richtlijn Osteoporose en Fractuurpreventie 2011. https:// www.volksgezondheidenzorg.info/bestanden/documenten/cborichtlijn-osteoporose-en-fractuurpreventie-2011. Accessed 2 Dec 2019

17. Genant HK, Wu CY, van Kuijk C, Nevitt MC (1993) Vertebral fracture assessment using a semiquantitative technique. J Bone Miner Res 8(9):1137-1148

18. Malgo F, Hamdy NA, Papapoulos SE, Appelman-Dijkstra NM (2017) Impact microindentation: consistency of serial measurements and alterations in patients with Paget's disease of the tibia. J Bone Miner Res 32(12):2375-2380

19. Malgo F, Hamdy NA, Rabelink TJ, Kroon HM, Claessen KM, Pereira AM et al (2017) Bone material strength index as measured by impact microindentation is altered in patients with acromegaly. Eur J Endocrinol 176(3):339-347

20. Furst JR, Bandeira LC, Fan WW, Agarwal S, Nishiyama KK, McMahon DJ, Dworakowski E, Jiang H, Silverberg SJ, Rubin MR (2016) Advanced glycation endproducts and bone material strength in type 2 diabetes. J Clin Endocrinol Metab 101(6): 2502-2510

21. Guerri-Fernandez R, Molina-Morant D, Villar-Garcia J, Herrera S, Gonzalez-Mena A, Guelar A et al (2017) Bone density, microarchitecture, and tissue quality after long-term treatment with tenofovir/emtricitabine or abacavir/lamivudine. J Acquir Immune Defic Syndr 75(3):322-327
22. Perez-Saez MJ, Herrera S, Prieto-Alhambra D, Vilaplana L, Nogues $\mathrm{X}$, Vera $\mathrm{M}$ et al (2017) Bone density, microarchitecture, and material strength in chronic kidney disease patients at the time of kidney transplantation. Osteoporos Int 28(9):2723-2727

23. Perez-Saez MJ, Herrera S, Prieto-Alhambra D, Nogues X, Vera M, Redondo-Pachon D et al (2017) Bone density, microarchitecture, and tissue quality long-term after kidney transplant. Transplantation. 101(6):1290-1294

24. Duarte Sosa D, Vilaplana L, Guerri R, Nogues X, Wang-Fagerland M, Diez-Perez A et al (2015) Are the high hip fracture rates among Norwegian women explained by impaired bone material properties? J Bone Miner Res 30(10):1784-1789

25. Sosa DD, Eriksen EF (2016) Women with previous stress fractures show reduced bone material strength: microindentation measurements in a retrospective case-control study of 60 subjects. Acta Orthop 87(6):626-631

26. Rufus-Membere P, Holloway-Kew KL, Diez-Perez A, Kotowicz MA, Pasco JA (2018) Feasibility and tolerability of bone impact microindentation testing: a cross-sectional, population-based study in Australia. BMJ Open 8(12):e023959

27. Popp KL, Caksa S, Martinez-Betancourt A, Yuan A, Tsai J, Yu EW et al (2019) Cortical bone material strength index and bone microarchitecture in postmenopausal women with atypical femoral fractures. J Bone Miner Res 34(1):75-82

28. Nogues X, Prieto-Alhambra D, Guerri-Fernandez R, Garcia-Giralt N, Rodriguez-Morera J, Cos L et al (2017) Fracture during oral bisphosphonate therapy is associated with deteriorated bone material strength index. Bone. 103:64-69

29. Paschalis EP, Gamsjaeger S, Klaushofer K (2017) Vibrational spectroscopic techniques to assess bone quality. Osteoporos Int 28(8): 2275-2291

30. Boskey AL (1992) Mineral-matrix interactions in bone and cartilage. Clin Orthop Relat Res (281):244-74

31. Donnelly E, Chen DX, Boskey AL, Baker SP, van der Meulen MC (2010) Contribution of mineral to bone structural behavior and tissue mechanical properties. Calcif Tissue Int 87(5):450-460

32. Boskey AL, Spevak L, Weinstein RS (2009) Spectroscopic markers of bone quality in alendronate-treated postmenopausal women. Osteoporos Int 20(5):793-800

33. Gourion-Arsiquaud S, Allen MR, Burr DB, Vashishth D, Tang SY, Boskey AL (2010) Bisphosphonate treatment modifies canine bone mineral and matrix properties and their heterogeneity. Bone. 46(3): 666-672

34. Gamsjaeger S, Buchinger B, Zwettler E, Recker R, Black D, Gasser JA et al (2011) Bone material properties in actively bone-forming trabeculae in postmenopausal women with osteoporosis after three years of treatment with once-yearly Zoledronic acid. J Bone Miner Res 26(1):12-18

35. Paschalis EP, Boskey AL, Kassem M, Eriksen EF (2003) Effect of hormone replacement therapy on bone quality in early postmenopausal women. J Bone Miner Res 18(6):955-959

36. Hassler N, Gamsjaeger S, Hofstetter B, Brozek W, Klaushofer K, Paschalis EP (2015) Effects of long-term alendronate treatment on postmenopausal osteoporosis bone material properties. Osteoporos Int 26(1):339-352

37. Dempster DW, Brown JP, Fahrleitner-Pammer A, Kendler D, Rizzo S, Valter I, Wagman RB, Yin X, Yue SV, Boivin G (2018) Effects of long-term denosumab on bone histomorphometry and mineralization in women with postmenopausal osteoporosis. J Clin Endocrinol Metab 103(7):2498-2509

Publisher's note Springer Nature remains neutral with regard to jurisdictional claims in published maps and institutional affiliations. 